EUROPEAN JOURNAL OF PURE AND APPLIED MATHEMATICS

Vol. 14, No. 4, 2021, 1402-1414

ISSN 1307-5543 - ejpam.com

Published by New York Business Global

\title{
Necessary Conditions for the Existence of a Saddle Point in one Optimal Control Problem for Systems of Hyperbolic Equations
}

Aysel Telman qizi Ramazanova

Department of Nichtlineare Optimierung, Faculty of Mathematic, University Duisburg-Essen, Essen, Germany,

\begin{abstract}
In this paper, we consider one optimal control problem with a multipoint quality functional described by a system of nonlinear hyperbolic equations with Goursat boundary conditions. Using a modified version of the increment method, various necessary first-order optimality conditions such as the Pontryagin maximum principle and the linearized maximum condition are proved.
\end{abstract}

2020 Mathematics Subject Classifications: 49k10, 49k20

Key Words and Phrases: Hyperbolic equation, necessary optimality condition, saddle point, increment method.

\section{Problem Statement.}

Let in a given rectangle $D=\left[t_{0}, t_{1}\right] \times\left[x_{0}, x_{1}\right]$ a controlled process described by the following system of nonlinear hyperbolic equations

$$
z_{t x}=f\left(t, x, z, z_{t}, z_{x}, u, v\right),(t, x) \in D
$$

with Goursat boundary conditions

$$
\begin{gathered}
z\left(t_{0}, x\right)=a(x), \quad x \in X=\left[x_{0}, x_{1}\right], \\
z\left(t, x_{0}\right)=b(t), \quad t \in T=\left[t_{0}, t_{1}\right], \\
a\left(x_{0}\right)=b\left(t_{0}\right) .
\end{gathered}
$$

Here $a(x), b(t)$ - given absolutely continuous $n$-dimensional vector functions, $f\left(t, x, z, z_{t}, z_{x}, u, v\right)$ - a given $n$-dimensional vector-function continuous in the set of variables together with partial derivatives with respect to $\left(z, z_{t}, z_{x}\right), u(t, x)$ and $v(t, x)-\mathrm{r}$ and $\mathrm{q}$-dimensional, respectively, measurable and bounded control vector-functions satisfying type inclusion constraints of the form

DOI: https://doi.org/10.29020/nybg.ejpam.v14i4.4135

Email address: .ramazanova@uni-due.de (A.Ramazanova) 


$$
\begin{aligned}
& u(t, x) \in U \subset R^{r}, \quad(t, x) \in D, \\
& v(t, x) \in V \subset R^{q}, \quad(t, x) \in D .
\end{aligned}
$$

Control functions $u(t, x), v(t, x)$ with the listed properties are called admissible controls.

It is further assumed that for each given admissible control $\left(u^{o}(t, x), v^{o}(t, x)\right)$, the Goursat - Darboux boundary-value problem (1) - (2) has a unique absolutely continuous solution $z^{o}(t, x)$ (in the sense of [1]-[3]. Various sufficient conditions for the existence of absolutely continuous solutions to the Goursat - Darboux problem (1) - (2) are found, for example, in [1]-[3].

On the solutions of the boundary value problem (1) - (2) generated by all possible admissible controls, we define a multi-point functional

$$
S(u, v)=\varphi\left(z\left(T_{1}, X_{1}\right), \ldots, z\left(T_{k}, X_{k}\right)\right) .
$$

$\left(T_{i}, X_{i}\right), i=\overline{1, k}\left(t_{0}<T_{1}<T_{2}<\ldots<T_{k} \leq t_{1}, x_{0}<X_{1}<X_{2}<\ldots<X_{k} \leq x_{1}\right)$ are given points, $\varphi\left(z_{1}, z_{2}, \ldots, z_{k}\right)$ is a given continuously differentiable scalar function.

Further, by $\|\alpha\|$ we denote the norm of the vector $\alpha=\left(\alpha_{1}, \ldots, \alpha_{n}\right)^{\prime}$ in the form $\|\alpha\|=\sum_{i=1}^{n}\left|x_{i}\right|$, the prime (') for vectors is the scalar product operation, and for matrices the transpose operation. Further, the value of $o\left(\alpha^{2}\right)$ means that $o\left(\alpha^{2}\right) / \alpha^{2} \rightarrow 0$ for $\alpha \rightarrow 0$.Obviously, functional (4) is defined for all sets $(u(t, x), v(t, x))$, for which the corresponding solution $z(t, x)=z(t, x, u, v)$ of the boundary value problem (1) - (2) is defined on the entire domain $D$.

Consider the following game problem.

Suppose that the control $u(t, x)$ is controlled by the side, which seeks to minimize the functional (1), and the control $v(t, x)$, the side $B$, which seeks to maximize the same functional.

Among all the sets $(u(t, x), v(t, x))$ on which the functional (3) is defined, find a set $\left(u^{o}(t, x), v^{o}(t, x)\right)$, such that

$$
S\left(u^{o}, v\right) \leq S\left(u^{o}, v^{o}\right) \leq S\left(u, v^{o}\right)
$$

for any, $(u(t, x), v(t, x)) \in U \times V,(t, x) \in D$.

A set (pair) $\left(u^{o}(t, x), v^{o}(t, x)\right)$ satisfying condition (5) is called the saddle point of functional (4) (see, for example, [4]-[5]).

In this paper, using the methodology, which is a generalization of the methodology [4][5], the necessary conditions for the existence of a saddle point are derived. (A necessary condition is the existence of a saddle point such as the Pontryagin maximum principle.)

\section{A necessary optimality condition such as the Pontryagin maximum principle for the existence of a saddle point.}

Suppose that $z^{o}(t, x)$ is a solution to the boundary value problem (1) - (2) corresponding to the pair $\left(u^{o}(t, x), v^{o}(t, x)\right)$ and $\bar{z}(t, x)=z^{o}(t, x)+\Delta z(t, x)$ is a solu- 
tion to the problem (1) - (2) corresponding to the $\operatorname{set}\left(\bar{u}(t, x)=u^{o}(t, x)+\Delta u(t, x)\right.$, $\left.\bar{v}(t, x)=v^{o}(t, x)+\Delta v(t, x)\right)$.

Then it is clear that the increment $\Delta z(t, x)$ state, $\left(z^{o}(t, x), y^{o}(t, x)\right)$ satisfies the conditions

$$
\begin{gathered}
\Delta z_{t x}(t, x)=f\left(t, x, \bar{z}(t, x), \bar{z}_{t}(t, x), \bar{z}_{x}(t, x), \bar{u}(t, x), \bar{v}(t, x)\right)- \\
-f\left(t, x, z^{o}(t, x), z_{t}^{o}(t, x), z_{x}^{o}(t, x), u^{o}(t, x), v^{o}(t, x)\right), \quad(t, x) \in D, \\
\Delta z\left(t_{0}, x\right)=\Delta a(x), \quad x \in\left[x_{0}, x_{1}\right] \\
\Delta z\left(t, x_{0}\right)=0, \quad t \in\left[t_{0}, t_{1}\right] .
\end{gathered}
$$

Let $\psi^{o}(t, x)$ be an arbitrary $n$-dimensional vector function. Multiplying both sides of relation (6) scalarly on the left by $\psi(t, x)$, and then integrating both sides of the resulting relation over the region $D$

$$
\begin{gathered}
\int_{t_{0}}^{t_{1}} \int_{x_{0}}^{x_{1}} \psi^{o^{\prime}}(t, x) \Delta z_{t x}(t, x) d x d t \\
=\int_{t_{0}}^{t_{1}} \int_{x_{0}}^{x_{1}} \psi^{o^{\prime}}(t, x)\left[f\left(t, x, z(t, x), z_{t}(t, x), z_{x}(t, x), \bar{u}(t, x), \bar{v}(t, x)\right)-\right. \\
\left.\quad-f\left(t, x, z^{o}(t, x), z_{t}^{o}(t, x), z_{x}^{o}(t, x), u^{o}(t, x), v^{o}(t, x)\right)\right] d x d t .
\end{gathered}
$$

Hereinafter, the prime for vectors is the scalar product operation, and for matrices it means the transpose operation.

We introduce an analog of the Hamilton - Pontryagin function in the form

$$
H\left(t, x, z, z_{t}, z_{x}, u, v, \psi^{o}\right)=\psi^{o^{\prime}} \cdot f\left(t, x, z, z_{t}, z_{x}, u, v\right) .
$$

It is easy to see that

$$
\int_{t_{0}}^{t_{1}} \int_{x_{0}}^{x_{1}} \psi^{o^{\prime}}(t, x) \Delta z_{t x}(t, x) d x d t=
$$

$$
\begin{aligned}
= & \int_{t_{0}}^{t_{1}} \int_{x_{0}}^{x_{1}}\left[\left(H\left(t, x, z(t, x), z_{t}(t, x), z_{x}(t, x), u(t, x), v(t, x), \psi^{o}(t, x)\right)-\right.\right. \\
& \left.-H\left(t, x, z^{o}(t, x), z_{t}^{o}(t, x), z_{x}^{o}(t, x), u(t, x), v(t, x), \psi^{o}(t, x)\right)\right)+ \\
& +\left(H\left(t, x, z^{o}(t, x), z_{t}^{o}(t, x), z_{x}^{o}(t, x), u(t, x), v(t, x), \psi^{o}(t, x)\right)-\right. \\
& \left.\left.-H\left(t, x, z^{o}(t, x), z_{t}^{o}(t, x), z_{x}^{o}(t, x), u^{o}(t, x), v^{o}(t, x), \psi^{o}(t, x)\right)\right)\right] .
\end{aligned}
$$


We write the increment of functional

$$
\begin{gathered}
\Delta S\left(u^{o}, v^{o}\right)=S(u, v)-S\left(u^{o}, v^{o}\right)= \\
=\varphi\left(z\left(T_{1}, X_{1}\right), \ldots, z\left(T_{k}, X_{k}\right)\right)-\varphi\left(z^{o}\left(T_{1}, X_{1}\right), \ldots, z^{o}\left(T_{k}, X_{k}\right)\right) .
\end{gathered}
$$

Given identity (9) from (10), we have

$$
\begin{gathered}
\Delta S\left(u^{o}, v^{o}\right)=S(u, v)-S\left(u^{o}, v^{o}\right)= \\
=\varphi\left(z\left(T_{1}, X_{1}\right), \ldots, z\left(T_{k}, X_{k}\right)\right)-\varphi\left(z^{o}\left(T_{1}, X_{1}\right), \ldots, z^{o}\left(T_{k}, X_{k}\right)\right)+ \\
+\int_{t_{0}}^{t_{1}} \int_{x_{0}}^{x_{1}} \psi^{o^{\prime}}(t, x) \Delta z_{t x}(t, x) d x d t- \\
-\int_{t_{0}} \int_{x_{0}}^{t_{1}}\left[\left(H\left(t, x, z(t, x), z_{t}(t, x), z_{x}(t, x), u(t, x), v(t, x), \psi^{o}(t, x)\right)-\right.\right. \\
\left.-H\left(t, x, z^{o}(t, x), z_{t}^{o}(t, x), z_{x}^{o}(t, x), u(t, x), v(t, x), \psi^{o}(t, x)\right)\right)+ \\
+\left(H\left(t, x, z^{o}(t, x), z_{t}^{o}(t, x), z_{x}^{o}(t, x), u(t, x), v(t, x), \psi^{o}(t, x)\right)-\right. \\
\left.\left.-H\left(t, x, z^{o}(t, x), z_{t}^{o}(t, x), z_{x}^{o}(t, x), u^{o}(t, x), v^{o}(t, x), \psi^{o}(t, x)\right)\right)\right] d x d t .
\end{gathered}
$$

We introduce the notation

$$
\begin{gathered}
\Delta_{u v} H[t, x] \equiv H\left(t, x, z^{o}(t, x), z_{t}^{o}(t, x), z_{x}^{o}(t, x), u(t, x), v(t, x), \psi^{o}(t, x)\right)- \\
-H\left(t, x, z^{o}(t, x), z_{t}^{o}(t, x), z_{x}^{o}(t, x), u^{o}(t, x), v^{o}(t, x), \psi^{o}(t, x)\right), \\
\Delta_{u} H[t, x] \equiv H\left(t, x, z^{o}(t, x), z_{t}^{o}(t, x), z_{x}^{o}(t, x), u(t, x), v^{o}(t, x), \psi^{o}(t, x)\right)- \\
-H\left(t, x, z^{o}(t, x), z_{t}^{o}(t, x), z_{x}^{o}(t, x), u^{o}(t, x), v^{o}(t, x), \psi^{o}(t, x)\right), \\
f_{u}[t, x] \equiv f_{z}\left(t, x, z^{o}(t, x), z_{t}^{o}(t, x), z_{x}^{o}(t, x), u^{0}(t, x), v^{o}(t, x)\right), \\
H_{u}[t, x] \equiv H_{u}\left(t, x, z^{o}(t, x), z_{t}^{o}(t, x), z_{x}^{o}(t, x), u^{o}(t, x), v^{o}(t, x), \psi^{o}(t, x)\right), \\
\Delta_{\bar{u} \bar{v}} f[t] \equiv f\left(t, x, z^{o}(t, x), z_{t}^{o}(t, x), z_{x}^{o}(t, x), \bar{u}(t, x), \bar{v}(t, x)\right)- \\
-f\left(t, x, z^{o}(t, x), z_{t}^{o}(t, x), z_{x}^{o}(t, x), u^{o}(t, x), v^{o}(t, x)\right) .
\end{gathered}
$$

Taking into account the introduced notation and using the Taylor formula the increment formula (11) of the quality functional (4) is represented as:

$$
\begin{gathered}
\Delta S\left(u^{o}, v^{o}\right)=\sum_{i=1}^{k} \frac{\partial \varphi^{\prime}\left(\bar{z}\left(T_{1}, X_{1}\right), \ldots, \bar{z}\left(T_{k}, X_{k}\right)\right)}{\partial z_{i}} \Delta z\left(T_{i}, X_{i}\right)+o_{1}\left(\left\|\Delta z\left(T_{i}, X_{i}\right)\right\|\right)+ \\
\quad+\int_{t_{0}}^{t_{1}} \int_{x_{0}}^{x_{1}} \psi^{o^{\prime}}(t, x) \Delta z_{t x}(t, x) d x d t-\int_{t_{0}}^{t_{1}} \int_{x_{0}}^{x_{1}} \Delta_{\bar{u}(t, x) \bar{v}(t, x)} H[t, x] d x d t-
\end{gathered}
$$




$$
\begin{gathered}
-\int_{t_{0}}^{t_{1}} \int_{x_{0}}^{x_{1}} H_{z}^{\prime}\left(t, x, z^{o}(t, x), z_{t}^{o}(t, x), z_{x}^{o}(t, x), u^{o}(t, x), v^{o}(t, x), \psi^{o}(t, x)\right) \Delta z(t, x) d x d t- \\
-\int_{t_{0}}^{t_{1}} \int_{x_{0}}^{x_{1}} H_{z_{t}}^{\prime}\left(t, x, z^{o}(t, x), z_{t}^{o}(t, x), z_{x}^{o}(t, x), u^{o}(t, x), v^{o}(t, x), \psi^{o}(t, x)\right) \Delta z_{t}(t, x) d x d t- \\
-\int_{t_{0}}^{t_{1}} \int_{x_{0}}^{x_{1}} H_{z_{x}}^{\prime}\left(t, x, z^{o}(t, x), z_{t}^{o}(t, x), z_{x}^{o}(t, x), u^{o}(t, x), v^{o}(t, x), \psi^{o}(t, x)\right) \Delta z_{x}(t, x) d x d t- \\
-\int_{t_{0}}^{t_{1}} \int_{x_{0}}^{x_{1}} \Delta_{\bar{u}(t, x) \bar{v}(t, x)} H_{z}^{\prime}[t, x] \Delta z(t, x) d x d t- \\
-\int_{t_{0}}^{t_{1}} \int_{x_{0}}^{x_{1}} \Delta_{u(t, x) v(t, x)} H_{z_{t}}^{\prime}[t, x] \Delta z_{t}(t, x) d x d t- \\
-\int_{t_{0}}^{t_{1}} \int_{x_{0}}^{x_{1}} \int_{x_{0}}^{t_{1}} \Delta_{o_{2}} \\
x_{x_{1}}
\end{gathered}
$$

We will deal with the transformation of individual terms in the increment formula (12). Taking into account the boundary conditions (2), we can write that

$$
\begin{gathered}
\Delta z(t, x)=\int_{t_{0}}^{t} \int_{x_{0}}^{x} \Delta z_{\tau s}(\tau, s) d s d \tau, \\
\Delta z_{t}(t, x)=\int_{x_{0}}^{x} \Delta z_{t s}(t, s) d s, \\
\Delta z_{x}(t, x)=\int_{t_{0}}^{t} \Delta z_{\tau x}(\tau, x) d \tau .
\end{gathered}
$$

From (13)is obtain

$$
\Delta z\left(T_{i}, X_{i}\right)=\int_{t_{0}}^{t_{1}} \int_{x_{0}}^{x_{1}} \alpha_{i}(t, x) \Delta z_{t x}(t, x) d x d t
$$

$\alpha_{i}(t, x), i=\overline{1, k}$ characteristic function of the domain $\left[t_{0}, T_{i}\right] \times\left[x_{0}, X_{i}\right], i=\overline{1, k}$. 
Using (15) we obtain

$$
\begin{gathered}
\sum_{i=1}^{k} \frac{\partial \varphi_{1}^{\prime}\left(z^{0}\left(T_{1}, X_{1}\right), \ldots, z^{0}\left(T_{k}, X_{k}\right)\right)}{\partial z_{i}} \Delta z\left(T_{i}, X_{i}\right)= \\
=\int_{t_{0}}^{t_{1}} \int_{x_{0}}^{x_{1}} \sum_{i=1}^{k} \alpha_{i}(t, x) \frac{\partial \varphi^{\prime}\left(z^{0}\left(T_{1}, X_{1}\right), \ldots, z^{0}\left(T_{k}, X_{k}\right)\right)}{\partial z_{i}} \Delta z_{t x}(t, x) d x d t .
\end{gathered}
$$

Further, using identities (12), applying the two-dimensional analogue of the Fubini formula, we arrive at the following relations:

$$
\begin{aligned}
& \int_{t_{0}}^{t_{1}} \int_{x_{0}}^{x_{1}} H_{z}^{\prime}\left(t, x, z^{o}(t, x), z_{t}^{o}(t, x), z_{x}^{o}(t, x), u^{o}(t, x), v^{o}(t, x), \psi^{o}(t, x)\right) \Delta z(t, x) d x d t= \\
& =\int_{t_{0}}^{t_{1}} \int_{x_{0}}^{x_{1}}\left[\int_{t_{0}}^{t_{1}} \int_{x_{0}}^{x_{1}} H_{z}^{\prime}\left(\tau, s, z^{o}(\tau, s), z_{t}^{o}(\tau, s), z_{x}^{o}(\tau, s), u^{o}(\tau, s), v^{o}(\tau, s), \psi^{o}(\tau, s)\right) d s d \tau\right] \Delta z_{t x}(t, x) d x d t, \\
& \int_{t_{0}}^{t_{1}} \int_{x_{0}}^{x_{1}} H_{z_{t}}^{\prime}\left(t, x, z^{o}(t, x), z_{t}^{o}(t, x), z_{x}^{o}(t, x), u^{o}(t, x), v^{o}(t, x), \psi^{o}(t, x)\right) \Delta z_{t}(t, x) d x d t= \\
& =\int_{t_{0}}^{t_{1}} \int_{x_{0}}^{x_{1}}\left[\int_{x_{0}}^{x_{1}} H_{z_{t}}^{\prime}\left(t, s, z^{o}(t, s), z_{t}^{o}(t, s), z_{x}^{o}(t, s), u^{o}(t, s), v^{o}(t, s), \psi^{o}(t, s)\right) d s\right] \Delta z_{t x}(t, x) d x d t, \\
& \int_{t_{0}}^{t_{1}} \int_{x_{0}}^{x_{1}} H_{z_{x}}^{\prime}\left(t, x, z^{o}(t, x), z_{t}^{o}(t, x), z_{x}^{o}(t, x), u^{o}(t, x), v^{o}(t, x), \psi^{o}(t, x)\right) \Delta z_{x}(t, x) d x d t= \\
& =\int_{t_{0}}^{t_{1}} \int_{x_{0}}^{x_{1}}\left[\int_{t_{0}}^{t_{1}} H_{z_{x}}^{\prime}\left(\tau, x, z^{o}(\tau, x), z_{t}^{o}(\tau, x), z_{x}^{o}(\tau, x), u^{o}(\tau, x), v^{o}(\tau, x), \psi^{o}(\tau, x)\right) d \tau\right] \Delta z_{t x}(t, x) d x d t .
\end{aligned}
$$

Taking into account the proven identities in the increment formula (12), we obtain the increment formula in the form:

$$
\begin{gathered}
\Delta S\left(u^{o}, v_{t_{0}}^{o t_{1}} \int_{x_{0}}^{x_{1}}\left[\sum_{i=1}^{k} \frac{\partial \varphi^{\prime}\left(z\left(T_{1}, X_{1}\right), \ldots, z\left(T_{k}, X_{k}\right)\right)}{\partial z_{i}} \alpha_{i}(t, x)\right] \Delta z_{t x}(t, x) d x d t+\right. \\
+\int_{t_{0}}^{t_{1}} \int_{x_{0}}^{x_{1}} \psi^{o^{\prime}}(t, x) \Delta z_{t x}(t, x) d x d t-
\end{gathered}
$$


A. T. Ramazanova / Eur. J. Pure Appl. Math, 14 (4) (2021), 1402-1414

$$
\begin{aligned}
& -\int_{t_{0}}^{t_{1}} \int_{x_{0}}^{x_{1}}\left[\int_{t_{0}}^{t_{1}} \int_{x_{0}}^{x_{1}} H_{z}^{\prime}\left(\tau, s, z^{o}(\tau, s), z_{t}^{o}(\tau, s), z_{x}^{o}(\tau, s), u^{o}(\tau, s), v^{o}(\tau, s), \psi^{o}(\tau, s)\right) d s d \tau\right] \Delta z_{t x}(t, x) d x d t- \\
& -\int_{t_{0}}^{t_{1}} \int_{x_{0}}^{x_{1}}\left[\int_{x_{0}}^{x_{1}} H_{z_{t}}^{\prime}\left(t, s, z^{o}(t, s), z_{t}^{o}(t, s), z_{x}^{o}(t, s), u^{o}(t, s), v^{o}(t, s), \psi^{o}(t, s)\right) d s\right] \Delta z_{t x}(t, x) d x d t- \\
& -\int_{t_{0}}^{t_{1}} \int_{x_{0}}^{x_{1}}\left[\int_{t_{0}}^{t_{1}} H_{z_{x}}^{\prime}\left(\tau, x, z^{o}(\tau, x), z_{t}^{o}(\tau, x), z_{x}^{o}(\tau, x), u^{o}(\tau, x), v^{o}(\tau, x), \psi^{o}(\tau, x)\right) d \tau\right] \Delta z_{t x}(t, x) d x d t- \\
& -\int_{t_{0}}^{t_{1}} \int_{x_{0}}^{x_{1}} \Delta_{u(t, x)}(t, x) H(t, x) d x d t-\int_{t_{0}}^{t_{1}} \int_{x_{0}}^{x_{1}} \Delta_{u(t, x)}(t, x) H_{z}^{\prime}(t, x) \Delta z(t, x) d x d t- \\
& -\int_{t_{0}}^{t_{1}} \int_{x_{0}}^{x_{1}} \Delta_{u(t, x) v(t, x)} H_{z_{t}}^{\prime}(t, x) \Delta z_{t}(t, x) d x d t-\int_{t_{0}}^{t_{1}} \int_{x_{0}}^{x_{1}} \Delta_{u(t, x) v(t, x)} H_{z_{x}}^{\prime}(t, x) \Delta z_{x}(t, x) d x d t+ \\
& +o_{1}\left(\sum_{i=1}^{k}\left\|\Delta z\left(T_{i}, X_{i}\right)\right\|\right)-\int_{t_{0}}^{t_{1}} \int_{x_{0}}^{x_{1}} o_{2}\left(\|\Delta z(t, x)\|+\left\|\Delta z_{t}(t, x)\right\|+\left\|\Delta z_{x}(t, x)\right\|\right) d x d t .
\end{aligned}
$$

Assuming that

$$
\begin{aligned}
\psi(t, x) & =\int_{t_{0}}^{t_{1}} \int_{x_{0}}^{x_{1}} H_{z}^{\prime}\left(\tau, s, z^{o}(\tau, s), z_{t}^{o}(\tau, s), z_{x}^{o}(\tau, s), u^{o}(\tau, s), v^{o}(\tau, s), \psi^{o}(\tau, s)\right) d s d \tau+ \\
& +\int_{x_{0}}^{x_{1}} H_{z_{t}}^{\prime}\left(t, s, z^{o}(t, s), z_{t}^{o}(t, s), z_{x}^{o}(t, s), u^{o}(t, s), v^{o}(t, s), \psi^{o}(t, s)\right) d s+ \\
+ & \int_{t_{0}}^{t_{1}} H_{z_{x}}^{\prime}\left(\tau, x, z^{o}(\tau, x), z_{t}^{o}(\tau, x), z_{x}^{o}(\tau, x), u^{o}(\tau, x), v^{o}(\tau, x), \psi^{o}(\tau, x)\right) d \tau,
\end{aligned}
$$

then the increment formula (17)takes the form

$$
\begin{gathered}
\Delta S\left(u^{o}, v^{o}\right)= \\
=-\int_{t_{0}} \int_{x_{0}}^{t_{1}} \Delta_{\bar{u}(t, x) \bar{v}(t, x)} H[t, x] d x d t-\int_{t_{0}}^{x_{1}} \int_{x_{0}}^{x_{1}} \Delta_{\bar{u}(t, x) \bar{v}(t, x)} H_{z}^{\prime}[t, x] \Delta z(t, x) d x d t- \\
-\int_{t_{0}}^{t_{1}} \int_{x_{0}}^{x_{1}} \Delta_{\bar{u}(t, x)} \bar{v}(t, x) H_{z_{t}}^{\prime}[t, x] \Delta z_{t}(t, x) d x d t- \\
-\int_{t_{0}}^{t_{1}} \int_{x_{0}}^{x_{1}} \Delta_{\bar{u}(t, x)}
\end{gathered}
$$




$$
-\int_{t_{0}}^{t_{1}} \int_{x_{0}}^{x_{1}} o_{2}\left(\|\Delta z(t, x)\|+\left\|\Delta z_{t}(t, x)\right\|+\left\|\Delta z_{x}(t, x)\right\|\right) d x d t .
$$

Relation (17) is called the adjoint system in the problem under consideration and is a linear two-dimensional Volterra-type integral equation with one-dimensional terms. It can be shown (for example, by the method of successive approximations) the existence of the uniqueness of a unique solution of the adjoint system in the class of measurable and bounded vector functions. The system of equations (18) is called the adjoint system. From the estimates established in [1]-[3] and others it follows that

$$
\begin{gathered}
\|\Delta z(t, x)\| \leq L_{1} \int_{t_{0}}^{t} \int_{x_{0}}^{x}\left\|\Delta_{\bar{u} \bar{v}} f[\tau, s]\right\| d s d \tau, \\
\left\|\Delta z_{t}(t, x)\right\| \leq L_{2}\left[\int_{t_{0}}^{t} \int_{x_{0}}^{x}\left\|\Delta_{\bar{u} \bar{v}} f[\tau, s]\right\| d s d \tau+\int_{x_{0}}^{x}\left\|\Delta_{\bar{u} \bar{v}} f[t, s]\right\| d s\right], \\
\left\|\Delta z_{x}(t, x)\right\| \leq L_{3}\left[\int_{t_{0}}^{t} \int_{x_{0}}^{x}\left\|\Delta_{\bar{u} \bar{v}} f[\tau, s]\right\| d s d \tau+\int_{t_{0}}^{t}\left\|\Delta_{\bar{u} \bar{v}} f[\tau, x]\right\| d \tau\right],
\end{gathered}
$$

$L_{i}=$ const $>0, i=\overline{1,3}$ some constants.

If we assume that $\left(u^{o}, v^{o}\right)$ is a saddle point, then from (5) we obtain that

$$
\begin{aligned}
& S\left(u^{o}+\Delta u, v^{o}\right)-S\left(u^{o}, v^{o}\right) \geq 0, \\
& S\left(u^{o}, v^{o}+\Delta v\right)-S\left(u^{o}, v^{o}\right) \leq 0 .
\end{aligned}
$$

Using relation (20), (21) and using formula (18) we arrive at the relations:

$$
\begin{gathered}
S\left(u^{o}+\Delta u, v^{o}\right)-S\left(u^{o}, v^{o}\right)= \\
=-\int_{t_{0}}^{t_{1}} \int_{x_{0}}^{x_{1}} \Delta_{u(t, x)} H(t, x) d x d t-\int_{t_{0}}^{t_{1}} \int_{x_{0}}^{x_{1}} \Delta_{u(t, x)} H_{z}^{\prime}(t, x) \Delta z(t, x) d x d t- \\
-\int_{t_{0}}^{t_{1}} \int_{x_{0}}^{x_{1}} \Delta_{u(t, x)} H_{z_{t}}^{\prime}(t, x) \Delta z_{t}(t, x) d x d t-\int_{t_{0}}^{t_{1}} \int_{x_{0}}^{x_{1}} \Delta_{u(t, x)} H_{z_{x}}^{\prime}(t, x) \Delta z_{x}(t, x) d x d t+ \\
+o_{1}\left(\sum_{i=1}^{k}\left\|\Delta z\left(T_{i}, X_{i}\right)\right\|\right)-\int_{t_{0}}^{t_{1}} \int_{x_{0}}^{x_{1}} o_{2}\left(\|\Delta z(t, x)\|+\left\|\Delta z_{t}(t, x)\right\|+\left\|\Delta z_{x}(t, x)\right\|\right) d x d t \\
S_{\left(u^{o}, v^{o}+\Delta v\right)-S\left(u^{o}, v^{o}\right)=}
\end{gathered}
$$




$$
\begin{gathered}
=-\int_{t_{0}}^{t_{1}} \int_{x_{0}}^{x_{1}} \Delta_{v(t, x)} H(t, x) d x d t-\int_{t_{0}}^{t_{1}} \int_{x_{0}}^{x_{1}} \Delta_{v(t, x)} H_{z}^{\prime}(t, x) \Delta z(t, x) d x d t- \\
-\int_{t_{0}}^{t_{1}} \int_{x_{0}}^{x_{1}} \Delta_{v(t, x)} H_{z_{t}}^{\prime}(t, x) \Delta z_{t}(t, x) d x d t-\int_{t_{0}}^{t_{1}} \int_{x_{0}}^{x_{1}} \Delta_{v(t, x)} H_{z_{x}}^{\prime}(t, x) \Delta z_{x}(t, x) d x d t+ \\
+o_{1}\left(\sum_{i=1}^{k}\left\|\Delta z\left(T_{i}, X_{i}\right)\right\|\right)-\int_{t_{0}}^{t_{1}} \int_{x_{0}}^{x_{1}} o_{2}\left(\|\Delta z(t, x)\|+\left\|\Delta z_{t}(t, x)\right\|+\left\|\Delta z_{x}(t, x)\right\|\right) d x d t .
\end{gathered}
$$

The obtained partial increment formulas (22), (23) of the quality functional allow us to prove the necessary optimality condition for the existence of a saddle point.

Let $(\theta, \xi) \in\left[t_{0}, t_{1}\right) \times\left[x_{0}, x_{1}\right)$ be an arbitrary regular point (Lebesgue point) (see, for example, [1],[2]; [8]) of the control $u^{o}(t, x), \varepsilon>0$ be a sufficiently small arbitrary number such that, $\theta+\varepsilon<t_{1}, \xi+\varepsilon<x_{1}$, and $u \in U$ and an arbitrary vector. The special control increment $u^{o}(t, x)$ is determined by the formula

$$
\Delta u(t, x ; \varepsilon)=\left\{\begin{array}{l}
u-u^{o}(t, x), \quad(t, x) \in D_{\varepsilon}=(\theta, \theta+\varepsilon) \times(\xi, \xi+\varepsilon), \\
0, \quad(t, x) \in D \backslash D_{\varepsilon} .
\end{array}\right.
$$

By $\Delta z(t, x ; \varepsilon)$ we denote the special state $z^{o}(t, x)$ corresponding to the increment $(24)$ of the $u^{o}(t, x)$ control.

Taking into account estimates (19), formula (24) for a special control increment, and also applying the mean value theorem from $(22)$, we obtain

$$
-\varepsilon^{2} \Delta_{u} H(\theta, \xi)+o\left(\varepsilon^{2}\right) \geq 0 .
$$

Consequently

$$
\Delta_{u} H(\theta, \xi) \leq 0 .
$$

Further, considering $\mu>0$ as an arbitrary sufficiently small number, if the special control increment $v^{o}(t, x)$ is determined by the formula

$$
\Delta v(t, x ; \mu)=\left\{\begin{array}{l}
v-v^{o}(t, x), \quad(t, x) \in D_{\mu}=(\theta, \theta+\mu) \times(\xi, \xi+\mu), \\
0, \quad(t, x) \in D \backslash D_{\mu},
\end{array}\right.
$$

where $\theta$ is an arbitrary vector, then from the increment formula (23) we similarly obtain that along the saddle point

$$
-\mu^{2} \Delta_{v} H(\theta, \xi)+o\left(\mu^{2}\right) \leq 0,
$$

Therefore, from inequality (25) it follows that

$$
\Delta_{v} H(\theta, \xi) \geq 0 .
$$

Given the arbitrariness of $u \in U, v \in V,(\theta, \xi) \in\left[t_{0}, t_{1}\right) \times\left[x_{0}, x_{1}\right)$ formulate the result. 
Theorem 1. If the set $\left(u^{o}(t, x), v^{o}(t, x)\right)$ is a saddle point of functional (4) under constraints (1) - (3). Then, with the necessity of the following conditions:

$$
\begin{aligned}
& \max _{u \in U} H\left(\theta, \xi, z^{o}(\theta, \xi), z_{t}^{o}(\theta, \xi), z_{x}^{o}(\theta, \xi), u, v^{o}(\theta, \xi), \psi^{o}(\theta, \xi)\right)= \\
= & H\left(\theta, \xi, z^{o}(\theta, \xi), z_{t}^{o}(\theta, \xi), z_{x}^{o}(\theta, \xi), u^{o}(\theta, \xi), v^{o}(\theta, \xi), \psi^{o}(\theta, \xi)\right),
\end{aligned}
$$

for all $u \in U$ and $(\theta, \xi) \in\left[t_{0}, t_{1}\right) \times\left[x_{0}, x_{1}\right)$,

$$
\begin{aligned}
& \min _{v \in V} H\left(\theta, \xi, z^{o}(\theta, \xi), z_{t}^{o}(\theta, \xi), z_{x}^{o}(\theta, \xi), u^{o}(\theta, \xi), v, \psi^{o}(\theta, \xi)\right)= \\
= & H\left(\theta, \xi, z^{o}(\theta, \xi), z_{t}^{o}(\theta, \xi), z_{x}^{o}(\theta, \xi), u^{o}(\theta, \xi), v^{o}(\theta, \xi), \psi^{o}(\theta, \xi)\right),
\end{aligned}
$$

for allv $\in V,(\theta, \xi) \in\left[t_{0}, t_{1}\right) \times\left[x_{0}, x_{1}\right)$.

(1) is an analogue of the Pontryagin maximum principle for the problem under consideration.

\section{The case of convex control domains.}

Suppose that the sets $U$ and $V$ are convex, and the vector- function $f\left(t, x, z, z_{t}, z_{x}, u, v\right)$ is continuous in the set of variables along with partial derivatives in $\left(z, z_{t}, z_{x}, u, v\right)$. Then, with arguments similar to those from $\mathrm{n}(2)$, we can prove the validity of the increment formula:

$$
\begin{gathered}
\Delta S\left(u^{o}, v^{o}\right)=S\left(u^{o}+\Delta u, v^{o}\right)-S\left(u^{o}, v^{o}\right)=-\int_{t_{0}}^{t_{1}} \int_{x_{0}}^{x_{1}} H_{u}^{\prime}(t, x) \Delta u(t, x) d x d t- \\
-\int_{t_{0}}^{t_{1}} \int_{x_{0}}^{x_{1}} H_{v}^{\prime}(t, x) \Delta v(t, x) d x d t+o_{1}\left(\sum_{i=1}^{k}\left\|\Delta z\left(T_{i}, X_{i}\right)\right\|\right)- \\
-\int_{t_{0}}^{t_{1}} \int_{x_{0}}^{x_{1}} o_{3}\left(\|\Delta z(t, x)\|+\left\|\Delta z_{t}(t, x)\right\|+\left\|\Delta z_{x}(t, x)\right\|+\|\Delta u(t, x)\|+\|\Delta v(t, x)\|\right) d x d t .
\end{gathered}
$$

Since $f\left(t, x, z, z_{t}, z_{x}, u, v\right)$ is continuously differentiable with respect to $(u, v)$, by analogy with (19) we can prove the validity of the estimates

$$
\|\Delta z(t, x)\| \leq L_{4} \int_{t_{0}}^{t_{1}} \int_{x_{0}}^{x_{1}}[\|\Delta u(\tau, s)\|+\|\Delta v(\tau, s)\|] d \tau d s,
$$




$$
\begin{aligned}
& \left\|\Delta z_{t}(t, x)\right\| \leq L_{5}\left[\int_{t_{0}}^{t_{1}} \int_{x_{0}}^{x_{1}}[\|\Delta u(\tau, s)\|+\|\Delta v(\tau, s)\|] d \tau d s+\int_{x_{0}}^{x_{1}}[\|\Delta u(t, s)\|+\|\Delta v(t, s)\|] d s\right], \\
& \left\|\Delta z_{x}(t, x)\right\| \leq L_{6}\left[\int_{t_{0}}^{t_{1}} \int_{x_{0}}^{x_{1}}[\|\Delta u(\tau, s)\|+\|\Delta v(\tau, s)\|] d \tau d s+\int_{t_{0}}^{t_{1}}[\|\Delta u(\tau, x)\|+\|\Delta v(\tau, x)\|] d \tau\right],
\end{aligned}
$$

$L_{4}, L_{5}, L_{6}$ some positive constants.

Let $\varepsilon \in[0,1]$ an arbitrary number, and $u(t, x) \in U,(t, x) \in D$ arbitrary admissible control.

Then the special increment of the admissible control $u^{o}(t, x)$ can be determined by the formula

$$
\Delta u(t, x ; \varepsilon)=\varepsilon\left[u(t, x)-u^{o}(t, x)\right] .
$$

Thus given the estimate (27), (28) increments of formula (26) we find that

$$
\begin{gathered}
S\left(u^{o}(t, x)+\Delta u(t, x), v^{o}(t, x)\right)-S\left(u^{o}(t, x), v^{o}(t, x)\right)= \\
=-\varepsilon \int_{t_{0}}^{t_{1}} \int_{x_{0}}^{x_{1}} H_{u}^{\prime}[t, x]\left(u(t, x)-u^{o}(t, x)\right) d x d t+o(\varepsilon) .
\end{gathered}
$$

Now the special increment of the admissible control $v^{o}(t, x)$ is determined by the formula

$$
\Delta v(t, x ; \mu)=\mu\left(v(t, x)-v^{0}(t, x)\right),
$$

where $v(t, x)$ arbitrary admissible control, and $\mu \in[0,1]$ arbitrary number.

Moreover, taking into account estimates (27)from (26), we obtain

$$
\begin{gathered}
S\left(u^{o}(t, x), v^{o}(t, x)+\Delta v(t, x ; \mu)\right)-S\left(u^{o}(t, x), v^{o}(t, x)\right)= \\
=-\mu \int_{t_{0}}^{t_{1}} \int_{x_{0}}^{x_{1}} H_{v}^{\prime}[t, x]\left(v(t, x)-v^{o}(t, x)\right) d x d t+o(\mu) .
\end{gathered}
$$

It follows from expansions $(26),(31)$ that if $\left(u^{0}(t, x), v^{0}(t, x)\right)$ is a saddle point in the problem under consideration, then

$$
\begin{aligned}
& \int_{t_{0}}^{t_{1}} \int_{x_{0}}^{x_{1}} H_{u}^{\prime}[t, x]\left(u(t, x)-u^{o}(t, x)\right) d x d t \leq 0, \\
& \int_{t_{0}}^{t_{1}} \int_{x_{0}}^{x_{1}} H_{v}^{\prime}[t, x]\left(v(t, x)-v^{o}(t, x)\right) d x d t \geq 0 .
\end{aligned}
$$

Thus proved 
Theorem 2. If $f\left(t, x, z, z_{t}, z_{x}, u, v\right)$ is continuously differentiable with respect to $\left(z, z_{t}, z_{x}, u, v\right)$, and the sets $U$ and $V$ are convex, then for the admissible control $\left(u^{o}(t, x), v^{o}(t, x)\right)$ to be the saddle point of the problem under consideration, it is necessary that relations (32), (33) hold for all $u(t, x) \in U,(t, x) \in D, v(t, x) \in V,(t, x) \in D$, respectively.

Inequalities (32), (33) are an analogue of the linearized integral maximum principle.

Using the results of the work, for example, [6], we can show that this result is equivalent to the following.

Theorem 3. Under the assumptions made, the saddle point $\left(u^{o}(t, x), v^{o}(t, x)\right)$ in the problem under consideration satisfies the relations

$$
\max _{u \in U} H_{u}^{\prime}[\theta, \xi] u=H_{u}^{\prime}[\theta, \xi] u^{0}(\theta, \xi), \min _{v \in V} H_{v}^{\prime}[\theta, \xi] u=H_{v}^{\prime}[\theta, \xi] v^{0}(\theta, \xi)
$$

for all $(\theta, \xi) \in\left[t_{0}, t_{1}\right) \times\left[x_{0}, x_{1}\right)$.

This result is an analogue of the pointwise linearized maximum principle. Note that, in the case of a nonsmooth quality functional, analogues of (2) and (3)will not be equivalent (see, for example, [9]). Note that in the case of openness of the control domain by similar reasoning, we can calculate the first and second variations of the quality functional and establish an analog of the Euler equation, as well as an analog of the Legendre - Clebsch condition.

\section{References}

[1] Plotnikov V.I., Sumin V.I, A ground-breaking achievement, The stability problem for nonlinear Goursat-Darboux systems // Differ. equations. (1972), No. 5, 845-856.

[2] Novozhenov M.M., Sumin V.I., Sumin M.I. A ground-breaking achievement, Methods of optimal control of mathematical physics systems. Gorky, Publishing House of the State University, (1986), 87 pp.

[3] Suryanarayana M.B. A ground-breaking achievement, Necessary optimality conditions for optimization problems with hyperbolic partial equations // SCAM, Journal Control.(1973), vol. 21, N3,130-137.

[4] Vasiliev F.P. Another fascinating Book, On the conditions for the existence of a saddle point of deterministic integro-differential games with delay in the presence of parameters // Zh. calc. mate. and mat. Physics, (1970), No. 1 p. 15-25.

[5] Vasiliev F.P. A very interesting paper, On the conditions for the existence of a saddle point in deterministic games for integro-differential systems with a delay of a neutral type // Automation and Telemechanics. (1972), No. 2, p. 40-50.

[6] Urgent V.A, Another fascinating Book, Computational methods of optimal control. Irkutsk, ISU Publishing House, (1982), 110 pp. 
[7] Gabasov R., Kirillova F.M. et al. Another fascinating Book, Optimization methods. Mn Four Quarter Publishing House. (2011), 472 p.

[8] Pontryagin L.S., Boltyansky V.G., Gamkrelidze R.V., Mishchenko E.F. Another fascinating Book, The mathematical theory of optimal processes. M. Science, (1986). $386 \mathrm{p}$.

[9] Demyanov V.F. Rubinov A.M. A ground-breaking achievement, Fundamentals of nonsmooth analysis and quasidifferential calculus. M. Science, (1990).

[10] Ramazanova A.T. A new theorem, On determining initial conditions of equations flexural-torsional vibrations of a bar. European Journal of Pure and Applied Mathematics 12 (1),(2019), pp. 25-38. 